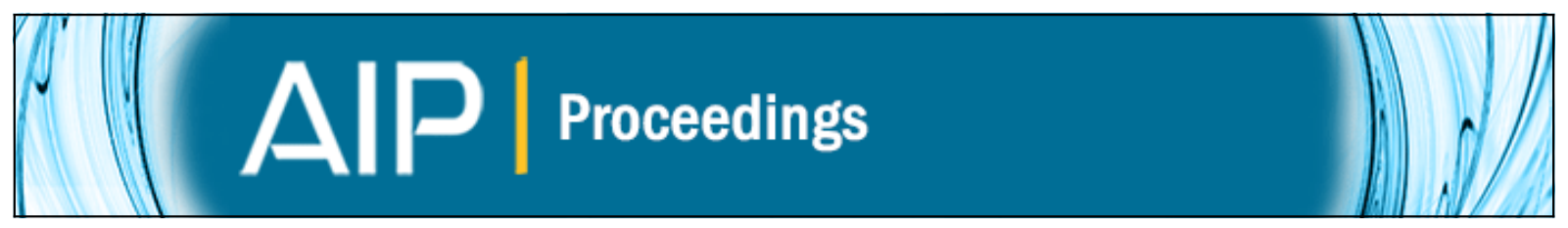

\title{
Parametric amplification of laser-driven acceleration in a plasma channel
}

Alexey V. Arefiev, Marius Schollmeier, and Vladimir N. Khudik

Citation: AIP Conference Proceedings 1507, 363 (2012); doi: 10.1063/1.4773723

View online: http://dx.doi.org/10.1063/1.4773723

View Table of Contents: http://scitation.aip.org/content/aip/proceeding/aipcp/1507?ver=pdfcov

Published by the AIP Publishing

Articles you may be interested in

Enhancement of laser-driven electron acceleration in an ion channel

Phys. Plasmas 21, 033104 (2014); 10.1063/1.4867491

Formation of a supersonic laser-driven plasma jet in a cylindrical channel

Phys. Plasmas 16, 114506 (2009); 10.1063/1.3270520

Laser-driven Acceleration in Clustered Plasmas

AIP Conf. Proc. 1086, 142 (2009); 10.1063/1.3080895

Progress of Laser-Driven Plasma Accelerators

AIP Conf. Proc. 920, 135 (2007); 10.1063/1.2756776

Laser-driven plasma-based accelerators: Wakefield excitation, channel guiding, and laser triggered particle injection

Phys. Plasmas 5, 1615 (1998); 10.1063/1.872820 


\title{
Parametric Amplification of Laser-Driven Acceleration in a Plasma Channel
}

\author{
Alexey V. Arefiev*, Marius Schollmeier ${ }^{\dagger}$ and Vladimir N. Khudik* \\ *Institute for Fusion Studies, The University of Texas, Austin, Texas 78712, USA \\ ${ }^{\dagger}$ Sandia National Laboratories, Albuquerque, New Mexico 87185, USA
}

\begin{abstract}
Two-dimensional particle-in-cell simulations are presented for a laser-irradiated solid-density target with and without an underdense preplasma. It is shown that an underdense preplasma can generate an energetic electron tail in addition to the warm electrons generated at the critical surface. Preplasma electrons are accelerated in a quasi-static positively charged channel formed by the laser. At ultra-relativistic laser intensities $\left(a_{0}=10\right)$, the acceleration mechanism is not sensitive to the laser polarization. An energetic tail with energies significantly exceeding the energy expected for a single electron in a vacuum is present in simulations with $s$ and $p$-polarized beams. This suggests that the mechanism of parametric amplification of laser-driven electron acceleration is a likely explanation for the observed phenomenon.
\end{abstract}

Keywords: laser-driven acceleration, betatron resonance, preplasma, parametric amplification, plasma channel PACS: $52.38 . \mathrm{Kd}, 41.75 . \mathrm{Jv}, 52.38 . \mathrm{Hb}$

\section{INTRODUCTION}

Sub-critical plasmas have an ability to generate relativistic energetic electrons when irradiated by a high-intensity laser beam. This feature has been demonstrated experimentally and it can be utilized in a range of applications, including Xray production [1], positron generation [2,3], and ion acceleration [4]. The electron acceleration mechanism depends significantly on laser pulse duration. For example, a laser-produced wakefield plays the dominant role when the beam is relatively short, with the duration shorter or comparable to the plasma wave period [5]. However, the wakefield mechanism becomes less efficient for longer laser pulses [6,7]. Our interest in such pulses is motivated by experiments with solid-density targets on fast proton generation with $1-5$ ps pulse duration $[8,9]$.

In these experiments, an intense laser beam irradiates a flat-foil solid-density target, generating a group of hot electrons at the front surface of the target. The hot electrons then spread inside the target and set up a sheath electric field at the front and back surfaces of the target. The sheath field confines the electrons inside the target, while at the same time it accelerates those ions that are located at the surface. The rear surface of the target is typically covered by a layer of contaminants, which contribute protons to an accelerating group of ions. The protons move ahead of the other ions because of their relatively high charge-to-mass ratio, generating an energetic proton beam at the rear surface of the target. The maximum and characteristic proton energies are determined by the electric field in the sheath. The amplitude of this field is directly dependent on the energy distribution of the hot electrons generated by the laser and, therefore, electron heating by the laser is a critical element in experiments aimed at generation of energetic proton beams.

One way to enhance electron energies is by increasing the intensity of the laser. This however introduces a new aspect that must be taken into consideration for systems with a significant prepulse. As the peak intensity is increased, the laser prepulse can become sufficient to preheat the target and alter its geometry. For example, a radiationhydrodynamics simulation for Sandia National Laboratories' Z-Petawatt prepulse parameters shows that a preheated target expands significantly, forming a transparent preplasma, many wavelengths thick, in front of the target [10]. Consequently, there are two spatially distinct areas of electron interaction with the main laser pulse if a significant prepulse is present: a steep boundary with a critical layer that absorbs and reflects the laser and an extended area of transparent preplasma in front of the target. The characteristic energy of the electrons accelerated at the critical surface is roughly equal to the electron oscillatory energy in the laser beam [11].

The extended interaction length in the preplasma is an advantage when it concerns the energy gain by electrons. Ideed, a free electron irradiated by an ultrarelativistic laser beam is pushed forward and most of its energy is associated with the axial motion rather than with the transverse oscillations. The maximum $\gamma$-factor that can be achieved by this electron is $\gamma=1+a_{0}^{2} / 2$, which significantly exceeds the $\gamma$-factor associated with the transverse oscillations, $\gamma_{\perp} \approx a_{0}$, 


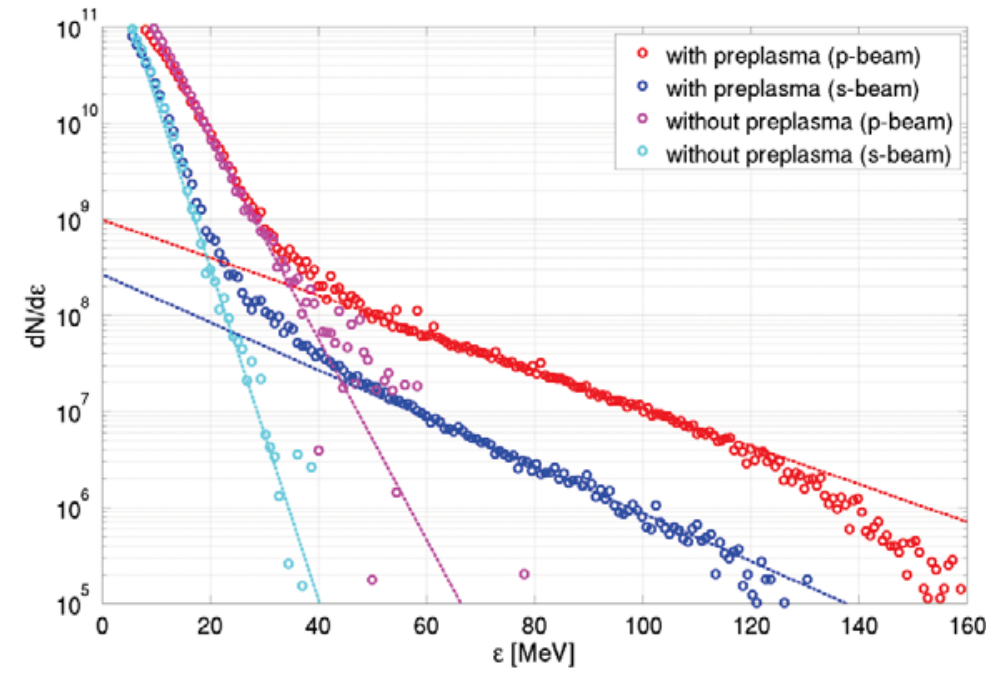

FIGURE 1. Electron spectra in targets with and without preplasma. The number of electrons is calculated assuming a width of 1 $\mu \mathrm{m}$ in the $x$ direction. The dashed lines are two-parameter Maxwellian fits $A \exp (-\varepsilon / T)$, where $A=8.69 \times 10^{11}$ and $T=2.52 \mathrm{MeV}$ for a target without preplasma irradiated by an $s$-polarized beam (cyan); $A=8.39 \times 10^{11}$ and $T=4.16 \mathrm{MeV}$ for a target without preplasma irradiated by a $p$-polarized beam (magenta); $A=2.65 \times 10^{8}$ and $T=17.47 \mathrm{MeV}$ for a target with preplasma irradiated by an $s$-polarized beam (blue); $A=9.79 \times 10^{8}$ and $T=22.12 \mathrm{MeV}$ for a target with preplasma irradiated by a $p$-polarized beam (red).

where $a_{0} \gg 1$ is the normalized laser amplitude. However, this requires an extended interaction length, because the electron has to travel roughly $\gamma$ wave-lengths with the beam in order to gain this energy. It is this paper, we examine laser interaction with an extended subcritical preplasma using two-dimensional particle-in-cell (PIC) simulations in order to understand if a similar mechanism can generate copious hot electrons in addition to the ones produced at the critical surface [11].

\section{2D PARTICLE-IN-CELL SIMULATION RESULTS}

In order to determine the role of the preplasma, we have performed two-dimensional particle-in-cell simulations using the Plasma Simulation Code (PSC) for a solid-density target with and without a preplasma. The main target is $250 \mu \mathrm{m}$ wide and $20 \mu \mathrm{m}$ thick, with the peak density $n_{0}=1.115 \times 10^{28} \mathrm{~m}^{-3}$. The preplasma is $250 \mu \mathrm{m}$ wide and $140 \mu \mathrm{m}$ thick, with the peak density $n_{p}=1.75 \times 10^{-3} n_{0}$. The electron density profile at the front side of the target $\left(z<z_{0}\right)$ is given by

$$
n_{e}=\left(\frac{n_{0}-n_{p}}{1+\exp \left[\left(\left|z-z_{0}\right|-w_{z}\right) / L_{z}\right]}+\frac{n_{p}}{1+\exp \left[\left(\left|z-z_{0}\right|-w_{p z}\right) / L_{z}\right]}\right) \frac{1}{1+\exp \left[\left(\left|y-y_{0}\right|-w_{y}\right) / L_{y}\right]},
$$

where $z_{0}=150 \mu \mathrm{m}, L_{z}=0.75 \mu \mathrm{m}, w_{z}=10 \mu \mathrm{m}, y_{0}=150 \mu \mathrm{m}, L_{y}=0.75 \mu \mathrm{m}, w_{y}=125 \mu \mathrm{m}$, and $w_{p z}=70 \mu \mathrm{m}$. The density profile at the rear side of the target is also given by Eq. (1) with $n_{p}=0$. The density profile for a target without the preplasma is also given by Eq. (1) where one must set $n_{p}=0$. The cell size in $(y, z)$ is $0.1 \mu \mathrm{m}$ by $0.1 \mu \mathrm{m}$, with each cell containing 9 electrons and 9 singly charged ions. We set the ion mass to be 1000 times the proton mass at the front of the target and in the preplasma in order to eliminate effects related to ion motion. The typical electron energies expected in the simulation are such that binary Coulomb collisions are negligible. We therefore turn off Coulomb collisions in PSC, which significantly speeds up the simulations and, most importantly, allows us to track individual energetic electrons.

The target is irradiated by a laser beam focused at the front side of the target, whose wavelength is $\lambda=1 \mu \mathrm{m}$, beam waist is $w_{0}=8 \mu \mathrm{m}$, and the normalized amplitude is $a_{0}=10$. The incidence angle is normal. In vacuum, the beam has a Gaussian shape in $y$ and a slowly varying envelope along $z$ (the corresponding characteristic scale $l$ is much longer 

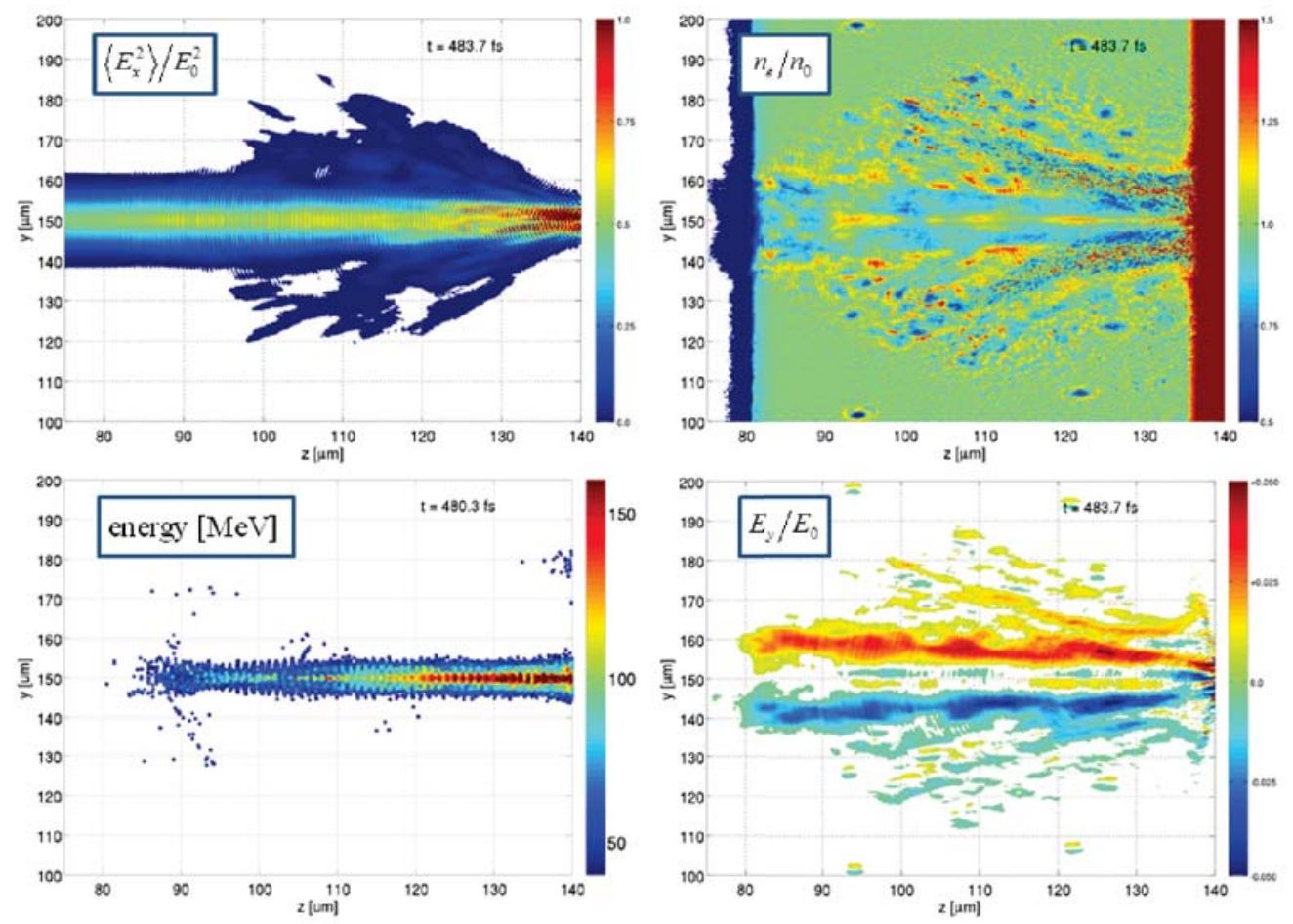

FIGURE 2. Snapshots of the electric field, electron density, and electron spectrum in a preplasma irradiated by an s-polarized beam.

than $\lambda$ ). The electric field of an $s$-polarized beam used in the simulations is given by

$$
E_{x}=E\left(z^{\prime}-c t\right)\left(1+\frac{z^{\prime 2}}{z_{R}^{2}}\right)^{-1 / 4} \exp \left[-\frac{y^{\prime 2} / w_{0}^{2}}{1+z^{\prime 2} / z_{R}^{2}}\right] \sin \left[\frac{2 \pi}{\lambda}\left(z^{\prime}-c t\right)+\frac{z^{\prime}}{z_{R}} \frac{y^{\prime 2} / w_{0}^{2}}{1+z^{\prime 2} / z_{R}^{2}}+\frac{1}{2} \cos ^{-1}\left(\frac{z^{\prime} / z_{R}}{\sqrt{1+z^{\prime 2} / z_{R}^{2}}}\right)\right]
$$

where $E$ is the envelope, $z_{R} \equiv \pi w_{0}^{2} / 2$ is the Rayleigh length, and $y^{\prime}=y-y_{0}$ and $z^{\prime}=z-z_{0}-w_{z}$ are the coordinates with the origin at the focal spot. Note that this expression defines a Gaussian beam with a constant focal spot as opposed to the expression for a 2D Gaussian beam given in [12]. The envelope in our simulations has a $160 \mu \mathrm{m}$ flat top and a rise length of $10 \mu \mathrm{m}$. The amplitude of the pulse $E_{0}$ is defined as $E_{0}=2 \pi a_{0} m_{e} c^{2} /|e| \lambda$, where $c$ is the speed of light and $m_{e}$ and $e$ are the electron charge and mass. The components of the magnetic field for the $s$-polarized wave follow from Maxwell's equations by substituting $E_{x}$ given by Eq. (2). In the case of a $p$-polarized wave, $E_{x}$ in Eq. (2) has to be simply replaced by $B_{x}$, with the components of the electric field $E_{y}$ and $E_{z}$ following from Maxwell's equations.

In the first two sets of simulations we computed the electron spectrum for the case of a target without a preplasma. The corresponding spectra for the $s$ and $p$-polarized beams and the corresponding Maxwellian fits are shown in Fig. 1. The $p$-polarized beam produces more energetic electrons than the $s$-polarized beam, but in both cases the temperature of the energetic electrons is below $5 \mathrm{MeV}$. We have then added the preplasma defined by Eq. (1) and calculated the resulting electron spectra for both beams, shown in Fig. 1. Each spectrum has a well pronounced energetic tail in addition to the warm population that was observed in the runs without the preplasma. The appearance of the energetic tails in the presence of the preplasma agrees well with the premise that the preplasma provides an extended interaction length for the electrons and therefore allows them to gain higher energies than during the interaction at the critical surface, where the axial interaction length is limited. However, a closer examination of the electron spectrum reveals that there are electrons with energies significantly higher than the maximum energy expected for a free electron in a vacuum, which is $25.5 \mathrm{MeV}\left(\gamma=1+a_{0}^{2} / 2\right)$ for a plane wave with $a_{0}=10$. 


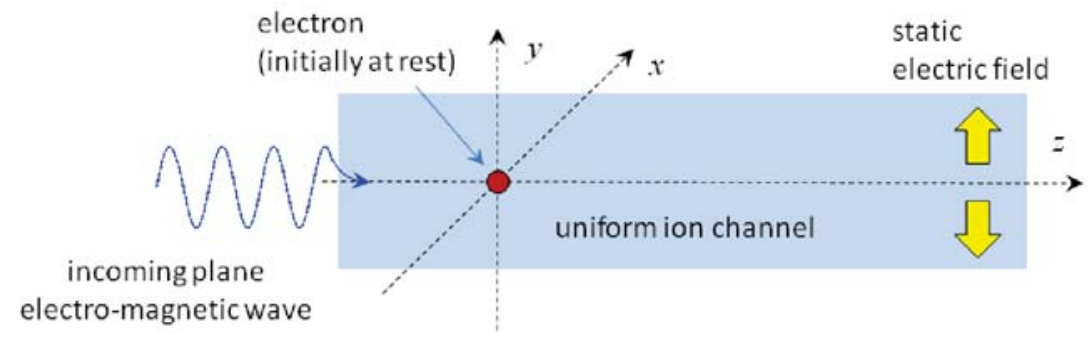

FIGURE 3. Schematic setup of the single-electron model.

A detailed look at the interaction of the laser beam with the preplasma reveals features not captured by the simple picture of a free electron in a vacuum. Figure 2 shows snapshots of $E_{x}^{2}$ and $n_{e}$ averaged over the laser period in the preplasma for the $s$-polarized beam. The ponderomotive pressure of the laser beam expels a significant number of plasma electrons from the beam in the transverse direction. The plasma mitigates this expulsion by generating a counteracting electric field $E_{y}$ via charge separation, shown in a lower-right panel of Fig. 2. As a result, the laser creates and maintains a positively charged channel with a quasi-static transverse electric field that evolves on an ion time scale. Since the ions are artificially heavy in our simulation, the evolution caused by their motion in the field of the channel is insignificant during the laser pulse. The formation of the channel is not sensitive to the polarization of the beam, so the quasi-static channel is present for both $s$ and $p$-polarizations.

We have tracked the energetic electrons from the tail of the distribution and found that the overwhelming majority of them have one unifying feature regardless of their initial position at the beginning of the simulation. These electrons at some stage become injected into the quasi-static channel at the edge of the preplasma. After that they begin to accelerate in the field of the laser and become confined in the channel. The electrons oscillate across the channel without leaving it while moving along with the laser beam. The laser is reflected after it reaches the critical layer, while the accelerated electrons are injected into the target.

A similar phenomenon at lower intensities has been previously demonstrated in particle-in-cell simulations [13]. However, the acceleration was only observed in the case of a $p$-polarized beam. The authors explained the observed phenomenon as a betatron resonance [13]: "An energetic electron experiences transverse betatron oscillations in the static fields. These oscillations are along the polarization of the laser pulse electric field, and thus an efficient energy exchange is possible when the betatron frequency is close to the laser frequency, as witnessed by the relativistic electron". This explanation implies that the laser field acts as a driving force for betatron oscillations, which requires proper polarization of the laser beam.

In contrast with that, our results show that the electron acceleration mechanism is not sensitive to the polarization of the laser at ultra-relativistic intensities $\left(a_{0}=10\right)$. The lower-left panel in Fig. 2 shows a scatter plot of electrons with energies above $45 \mathrm{MeV}$ in the preplasma. All of the electron are located inside the channel formed by the $s$-polarized laser beam. Since the laser is $s$-polarized, its electric field is pointing in/out of the plane of the plot. There is no driving force for the betatron oscillations in this case, which eliminates the betatron resonance as a mechanism responsible for the electron acceleration. On the other hand, a previously overlooked mechanism reported recently in Ref. [14] can explain both the observed energy gain and lack of sensitivity to the polarization at ultra-relativistic intensities. In the next section we give a brief overview of the mechanism and summarize the key findings.

\section{PARAMETRIC AMPLIFICATION OF BETATRON OSCILLATIONS}

In order to discuss the mechanism responsible for the enhancement of electron acceleration in an $s$-polarized beam, we consider the behavior of a single electron placed in a straight uniform ion channel and irradiated by a plane electromagnetic wave, as show in Fig. 3. The ions are assumed to be immobile. We choose a two-dimensional spatial setup $(y ; z)$, with the $y$-axis directed across the channel and the $z$-axis directed along the channel axis. The plane wave propagates in the positive direction along the $z$-axis. The electric and magnetic fields $(\mathbf{E}$ and $\mathbf{B})$ acting on the electron are thus given and the problem reduces to the analysis of the electron equations of motion,

$$
\frac{d \mathbf{r}}{d t}={ }_{\gamma}^{c} \mathbf{P}
$$




$$
\frac{d \mathbf{P}}{d t}=-\frac{|e| \mathbf{E}}{m_{e} c}-\frac{|e|}{\gamma m_{e} c} \mathbf{P} \times \mathbf{B}
$$

where $\mathbf{r}$ is the electron position, $t$ is the time in the laboratory (ion) frame of reference, $e$ and $m_{e}$ are the electron charge and mass, $c$ is the speed of light, $\mathbf{P}$ is the dimensionless electron momentum normalized to $m_{e} c$, and $\gamma=\sqrt{1+P^{2}}$ is the relativistic factor.

The electric field, $\mathbf{E}=\mathbf{E}_{\text {ion }}+\mathbf{E}_{\text {wave }}$, is a sum of a static field of the ion space-charge, $\mathbf{E}_{\text {ion }}$, and an oscillating field of the wave, $\mathbf{E}_{\text {wave }}$. The static component in the uniform channel is $\mathbf{E}_{i o n}=\mathbf{e}_{y} \omega_{p 0}^{2} m_{e} y /|e|$, where $\mathbf{e}_{y}$ is a unit vector, $\omega_{p 0} \equiv \sqrt{4 \pi n_{0} e^{2} / m_{e}}$ is the plasma frequency, and $n_{0}$ is the density of the singly charged ions in the channel. The magnetic field, $\mathbf{B}=\mathbf{B}_{\text {wave }}$, is only due to the wave. It is convenient to express the wave field in terms of a dimensionless vector potential a:

$$
\mathbf{E}_{\text {wave }}=-\frac{m_{e} c}{|e|} \frac{\partial \mathbf{a}}{\partial t}, \quad \mathbf{B}_{\text {wave }}=\frac{m_{e} c^{2}}{|e|} \nabla \times \mathbf{a}
$$

The vector potential a depends on $t$ and $z$ only via a phase variable

$$
\xi \equiv \omega(t-z / c)
$$

where $\omega$ is the frequency of the laser. We consider an s-polarized wave, so that $\mathbf{a}=a(\xi) \mathbf{e}_{x}$, where $\mathbf{e}_{x}$ is a unit vector and $a(\xi)$ is a sinusoidal function with a slowly varying envelope. For simplicity, we assume that $a(\xi)=a_{0} \sin (\xi)$ after some initial rise time, where $a_{0}$ is the maximum amplitude.

As shown in Ref. [14], transverse oscillation of the electron across the channel is described by a general secondorder nonlinear differential equation that follows directly from Eqs. (3) and (4). This equation reduces to a linear equation for an oscillator with a modulated eigenfrequency if the transverse displacement is small compared to $c / \omega_{p 0}$ :

$$
\frac{d^{2} y}{d \xi^{2}}+\frac{\omega_{p 0}^{2}}{\omega^{2}}\left[1+\frac{a_{0}^{2}}{2} \sin ^{2}(\xi)\right] y=0
$$

A clear advantage of using the phase variable $\xi$ as an independent variable instead of time $t$ is that the frequency of the modulations is constant (it is equal to unity). If the wave amplitude is non-relativistic $\left(a_{0} \ll 1\right)$, then the second term in the square brackets is negligible and the eigenfrequency is equal to $\omega_{p 0} / \omega$. We are interested in a regime where the plasma is underdense, $\omega_{p 0} \ll \omega$. In this case, the eigenfrequency is significantly lower than the frequency of the modulations, which makes the oscillations across the channel stable at non-relativistic intensities.

The conditions for instability become much more favorable at ultra-relativistic intensities $\left(a_{0} \gg 1\right)$. In this case, the first-term in the square brackets in Eq. (7) is negligible and the equation reduces to

$$
\frac{d^{2} y}{d \xi^{2}}+\frac{a_{0}^{2}}{2} \frac{\omega_{p 0}^{2}}{\omega^{2}} \sin ^{2}(\xi) y=0 .
$$

In contrast to the non-relativistic case, the eigenfrequency is now strongly modulated and, as a result, its maximum value increases with $a_{0}$ for a given ion density. Equation (8) is a Mathieu differential equation. A conventional stability analysis shows that its solutions are stable for $\omega_{p 0} a_{0} / \omega \ll 1$. Its solutions become unstable if the parameter $\omega_{p 0} a_{0} / \omega$ exceeds the instability threshold $\omega_{p 0} a_{0} / \omega \approx 1.62$ [14]. In contrast with the betatron resonance [13], this parametric instability of transverse oscillations is caused by the eigenfrequency modulations.

The parametric amplification of the transverse oscillations decreases the dephasing between the electron and the wave. Indeed, there is an integral of motion for the electron in the considered problem, $\gamma-P_{z}+\omega_{p 0}^{2} y^{2} / 2 c^{2}=$ const. Since the axial dephasing rate is $\gamma-P_{z}$, it will decrease as the amplitude of transverse oscillations increases. As a result, the electron stays in phase with the wave for a longer time and thereby gains more energy. It is shown in Ref. [14] that this mechanism of axial electron acceleration caused by the parametric instability works regardless of the polarization. The resulting maximum $\gamma$-factor is higher for a $p$-polarized wave, which implies that a $p$-polarized beam should produce electrons with higher energies than an $s$-polarized beam. These observations are consistent with the spectra shown in Fig. 1, which leads us to conclude that the mechanism of parametric amplification of laser-driven electron acceleration proposed in Ref. [14] is a likely cause of the electron acceleration observed in our 2D PIC simulations. 


\section{SUMMARY AND CONCLUDING REMARKS}

Our 2D PIC simulations of a target with and without preplasma show that an underdense preplasma can generate an energetic electron tail in addition to the warm electrons generated at the critical surface. The electrons are accelerated in a quasi-static positively charged channel formed by the laser. The acceleration mechanism is not sensitive to the polarization of the beam, which suggests that the mechanism proposed in Ref. [14] is a likely explanation for the observed phenomenon.

Considerable acceleration of electrons that has been previously observed experimentally in underdense plasmas has similar features $[1,2,15,16,17,18]$. It is therefore plausible that the mechanism of parametric amplification of laser-driven electron acceleration is involved in formation of energetic electron tails in these experiments. However, a realistic modeling of the experiments is required for a conclusive assessment. The amplification of electron oscillations via the presented mechanism may also be beneficial in those applications that employ transverse electron oscillations in a confining quasi-static field to generate $\mathrm{x}$ - and gamma-rays [17, 19], provided that the mechanism is robust with respect to spectral broadening of the laser beam [20]. The instability threshold in the single-electron model is determined by a product $\omega_{p 0} a_{0} / \omega$. Consequently, the plasma density threshold decreases with laser intensity and the instability can develop even in a very underdense plasma. On the other hand, the channel might become completely devoid of electrons at low densities. It is, therefore, important to determine a self-consistent instability threshold by considering both electron acceleration and channel formation. Finally, electron pre-acceleration during the injection into the channel provides the electron with initial axial momentum. As shown in Ref. [14], finite initial axial momentum lowers the instability threshold. The maximum $\gamma$-factor achieved by the electron increases if the electron is initially moving in the same direction as the laser beam with relativistic momentum. That is possibly why the maximum electron energies observed in our simulations exceed the energies predicted by the single-electron model. A simulation using test particles in a self-consistently generated channel might be useful to clarify this aspect.

\section{ACKNOWLEDGMENTS}

The simulations were performed using the Plasma Simulation Code (PSC), which was provided to the authors by Dr. H. Ruhl and Dr. A. Kemp. The authors acknowledge the Texas Advanced Computing Center at The University of Texas at Austin for providing HPC resources that have contributed to the research results reported within this paper. This work was supported by Sandia National Laboratory Contract No. PO 990947, National Nuclear Security Administration Contract No. DE-FC52-08NA28512, and U.S. Department of Energy Contract No. DE-FG02-04ER54742. Sandia is operated by Lockheed Martin Corp. for the U.S. DOE NNSA under contract No. DE-AC04-94AL85000.

\section{REFERENCES}

1. S. Kneip, C. McGuffey, S. R. Nagel ,et al., SPIE Proc. 7359, 73590T (2009).

2. C. Gahn, G. D. Tsakiris, G. Pretzler, et al., Phys. Plasmas 9, 987 (2002).

3. H. Chen, S. C. Wilks, D. D. Meyerhofer, et al., Phys. Rev. Lett. 105, 015003 (2010).

4. M. Roth, T. E. Cowan, M. H. Key, et al., Phys. Rev. Lett. 86, 436 (2001).

5. E. Esarey, C. B. Schroeder, and W. P. Leemans, Rev. Mod. Phys. 81, 1229 (2009).

6. V. Malka, J. Faure, J. R. Marques, et al., Phys. Plasmas 8, 2605 (2001).

7. S.P.D. Mangles, B. R. Walton, M. Tzoufras, et al., Phys. Rev. Lett. 94, 245001 (2005).

8. J. Fuchs, P. Antici, E. d'Humieres, et al., Nat. Phys. 248 (2006).

9. K. Flippo, T. Bartal, F. Beg, et al., Journal of Physics: Conference Series 244, 022033 (2010)

10. Private communications with Adam Sefkow at Sandia National Laboratories.

11. S. C. Wilks, W. L. Kruer, M. Tabak, and A. B. Langdon, Phys. Rev. Lett. 69, 1383 (1992).

12. J. Alda, "Laser and Gaussian beam propagation and transformation," in Encycl. of Opt. Engineering (Dekker, 2003$)$, p. 999.

13. A. Pukhov, Z.-M. Sheng, and J. M. ter Vehn, Phys. Plasmas 6, 2847 (1999).

14. A. V. Arefiev, B. N. Breizman, M. Schollmeier, and V. N. Khudik, Phys. Rev. Letters 108, 145004 (2012).

15. C. Gahn, G. D. Tsakiris, A. Pukhov, et al., Phys. Rev. Lett. 83, 4772 (1999).

16. Y. Kitagawa, Y. Sentoku, S. Akamatsu, et al., Phys. Rev. Lett. 92, 205002 (2004).

17. S. Kneip, S. R. Nagel, C. Bellei, et al., Phys. Rev. Lett. 100, 105006 (2008).

18. B. R. Walton, S. P. D. Mangles, Z. Najmudin, et al., Phys. Plasmas 13, 113103 (2006).

19. S. Cipiccia, M. R. Islam, B. Ersfeld, et al., Nature Phys. 7, 867 (2011).

20. S.Y. Kalmykov, A. Beck, S. A. Yi, et al., Phys. Plasmas 18, 056704 (2011). 